\title{
Automatic Pest Identification for Cotton Crop Using Convolution Neural Network
}

\author{
M. Kalpana ${ }^{1 *}$, K. Senguttuvan ${ }^{2}$ and P. Latha ${ }^{3}$ \\ ${ }^{1}$ Computer Science, Department of Social Science, ADAC\&RI, Tiruchirappalli, India \\ ${ }^{2}$ Entomology and ${ }^{3}$ Pathology, Department of Cotton, Coimbatore \\ Tamil Nadu Agricultural University, Tamil Nadu, India \\ *Corresponding author
}

\section{Keywords}

Bacterial blight, Anthracnose,

Leafhopper,

Convolutional neural networks,

Deep learning,

Cotton plant disease

Article Info

Accepted:

17 August 2020

Available Online:

10 September 2020

A B S T R A C T

Deep learning is the modern technique used for image processing. Particularly Convolutional Neural Networks (CNNs), is used for automatic identification of cotton Plant disease. Disease in cotton can interfere with the production of cotton and makes a distress to the country's economy. To manage the disease in cotton, an accurate diagnosis is essential. In this paper, to diagnosis the disease in cotton a research was carried out to automatically identify the cotton plant disease using Convolutional Neural Networks. Dataset with around 13,372 images for three diseases such as Bacterial Blight, Anthracnose, and Leafhopper are collected from cotton field. Convolutional Neural Networks is used to for both recognition and classification of three cotton disease images captured from farmer's field. The experimental result showed affirmative output of approximately 93.89 $\%$ accuracy of recognition of cotton plant disease using python programming.

\section{Introduction}

Cotton crop is cultivated for its fiber, which is unanimously used as a textile raw material. Cotton crop is an essential commodity in the world economy. So the cotton plant health is very much essential and closely linked with world economy. Food and Agriculture Organization (FAO) gave an estimate that the pests and diseases which leads to the loss of $20-40 \%$ of worldwide food production, constituting a hazard to food security (Food and Agriculture Organization of the United Nation, International Plant Protection Convention, 2017). Use of pesticides to cotton plant is the way of protecting crop from infestation and to maintain the yields. Applying the harmfully pesticides impacts 
biodiversity, including fish, insect and bird populations, and also water, soil and air quality (Risebrough, 1986; Goulson, 2014; Gill and Garg, 2014 Sanchez-Bayo and Goka, 2014; Knillmann and Liess, 2019). The use of pesticide also constitutes a risk to human health with both chronic and acute effects (Weisenburger, 1993; Bassil et al., 2007; Kim et al., 2016).

Farmers need knowledge regarding the proper practices in right time. Assessing the healthiness of the field and cotton crop is not simple and it requires a high level experts. Cotton disease can be expressed differently from one plant species to another or even from one variety to another. Assessing the healthiness of cotton plant disease is time consuming in large farms. The automatics identification of cotton plant disease by images is highly potential to solve the issues with expert assistance tools.

Convolutional Neural Networks (CNNs) is very successful tool in computer vision tasks, such as object recognition, detection biometry and classification. CNN can match filters that are directly derived from the data. The first research was used with CNN to classify and identify plant disease and the performance of the model is $96.3 \%$ for 13 different types of plant disease with healthy leaves to differentiate the leaves from its surroundings (Sladojevic et al., 2016).

CNN model was based on single classifier which is developed for detecting plant diseases for plantation. CNN was used to get top 5 errors of $16.4 \%$ for image classification with 1000 category of data classes (Alex K, 2012). CNN architecture was proposed to classify different types of plants from the images. The results obtained are compared with SVM classifiers with different kernels. The performance of $\mathrm{CNN}$ is $97.47 \%$ compared with the other methods (Hulya Yalcin et al., 2016). CNN was used to classify agricultural pest insects with saliency map with automated visual system which provides expert level pest insect recognition with the minimum of operational training (Z. Liu et al., 2016). Deep learning Convolutional Neural Networks is a significant technique to process the image. Review was conducted with 19 applications that relied on CNNs which automatically identify crop disease. CNN plays an important expertise assistance or automatic screening tool (Justine Boulent et al., 2019).

\section{Materials and Methods}

The main purpose of the articles is automatically identifying the cotton disease using images which helps to assess during the farming activities. This paper is organized as follows. The deep learning convolutional neural networks applied to cotton diseases identification is detailed in section 2. After describing $\mathrm{CNN}$, section 3 presents the experimental results. In section 4, we focus on CNN performance. Finally, in section 5, the conclusion and future research directions are also proposed.

\section{Deep learning convolutional neural networks applied to cotton diseases identification}

\section{Cotton disease diagnosis}

A Convolutional Neural Network is the base for computer vision technologies. Convolutional Neural Networks is similar to that of neural networks, made up of neurons with learnable weights and biases.

Each neuron in network receives several inputs, takes a weighted sum over them, pass it through an activation function and responds with an output. CNN uses two operations such as 'convolution' and pooling' to reduce an image into its essential features and uses those features to understand and classify the 
image. The $\mathrm{CNN}$ requires images to create the model.

\section{Algorithm for cotton disease identification}

\begin{tabular}{|l|l|}
\hline Step 1 & $\begin{array}{l}\text { Collect the image from cotton } \\
\text { field. }\end{array}$ \\
\hline Step 2 & $\begin{array}{l}\text { Process the image using image } \\
\text { enhancement techniques ie., } \\
\text { Unmask Sharpening Technique }\end{array}$ \\
\hline Step 3 & $\begin{array}{l}\text { Images are split into training } \\
(80 \%) \text { and test(20\%) }\end{array}$ \\
\hline Step 4 & $\begin{array}{l}\text { Convolution neural network is } \\
\text { used to create generate the model } \\
\text { using python programming }\end{array}$ \\
\hline
\end{tabular}

\section{Dataset}

The inputs for the Convolutional Neural Networks are the image collected from cotton field. The images related to leaf disease in cotton. They are

Leaf downward cupping (Insect: Leafhopper)

Leaf angular spot (Disease: Angular leaf spot)

Leaf burned appearance (Disease: Anthracnose)

\section{Cotton image enhancement}

The collected image from the field is processed with image enhancement techniques i.e., Unsharp Masking technique for leaf symptoms using MATLAB - Image Processing ToolBox.

Images are split into training $(80 \%)$ and test $(20 \%)$

The enhanced images are used for training and test the convolution neural network model. $80 \%$ of the data are taken as training and $20 \%$ are used for testing the model. Figure 1, 2, 3 shows the images of training and test images of bacterial blight, anthracnose and leaf hopper leaf images. Number of images taken for leaf diseases are 13,372. The training images are 11,042 and test images are 2330 .

\section{Architecture of convolutional neural networks for cotton disease diagnosis}

The architecture of model for a CNN is shown in Figure 4; the model is configured to take a fixed sized image of $128 \times 128$ RGB as an input for processing. All the training images are immediately center normalized and the CNN has 16 layers of the filters respectively. The learning rate $\mathrm{LR}=1 \mathrm{e}-4$. The model consists of the pooling layers and the fully connected layers.

Convolution layer: Convolution layers have a set of learnable filters. Each filter has small width and height and the same depth as that of 3 input layers as image input (three cotton leaf disease). The image size is set to 128 .

Activation layer (Relu): from the convolution layer a matrix is generates that is smaller in size than the original image. The generated matrix runs through an activation layer. The activation layer introduces non linearity to allow the network train itself with back propagation.

Pooling layer: pooling layer reduces the size of matrix. A filter is passed over the result obtained from the previous layer and selects one number out of each group of values. The max pooling is used which helps the network to train faster and focus on the important features of the leaf images.

Fully connected layer: This layer adopts the features of multilayer perceptron structure. The input obtained is one-dimensional vector representing the output of the previous layer. Its output is the list of probabilities of different labels attached with the image (e.g. bacterial blight, anthracnose and leaf hopper). 
The label with the highest probability is the classification label.

\section{Results and Discussion}

The collected images are processed with MATLAB Image Processing Toolbox to sharpen the images and latter the images are split into training and testing. With the training and testing images the $\mathrm{CNN}$ is constructed. In order to verify the reliability of cotton disease using convolution neural networks, a 16 layer convolution neural network is constructed, including the input layer, five convolution layers, five pooling layers, two local contrast adjustment layers, two full connection layers and one output layer using python 3.8.0. The training and test images of bacterial blight, anthracnose and leaf hopper are taken as the input images for the input layers. The images pass through all the layers in the $\mathrm{CNN}$ and the model is generated as shown in the Figure 5. With the generated model the accuracy of the model is tested. The optimizer used is 'adam', learning_rate is also used and the loss is 'categorical_crossentropy
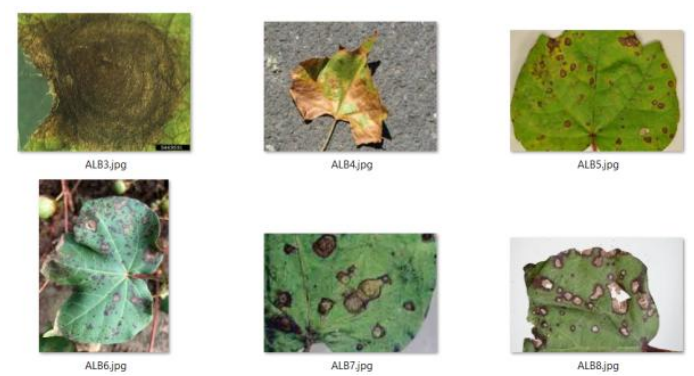

Figure.1 Images of Bacterial Blight(training and test image)
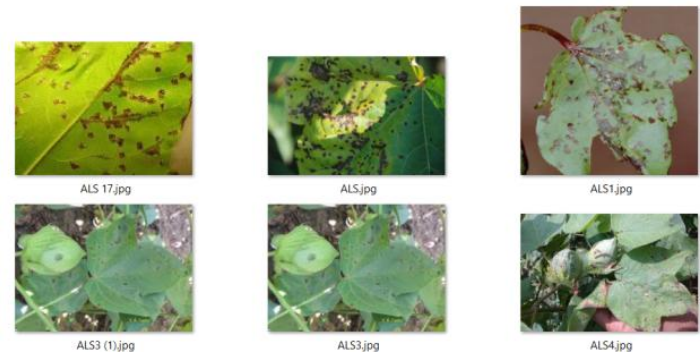

Figure.2 Images of Anthracnose (training and test image)
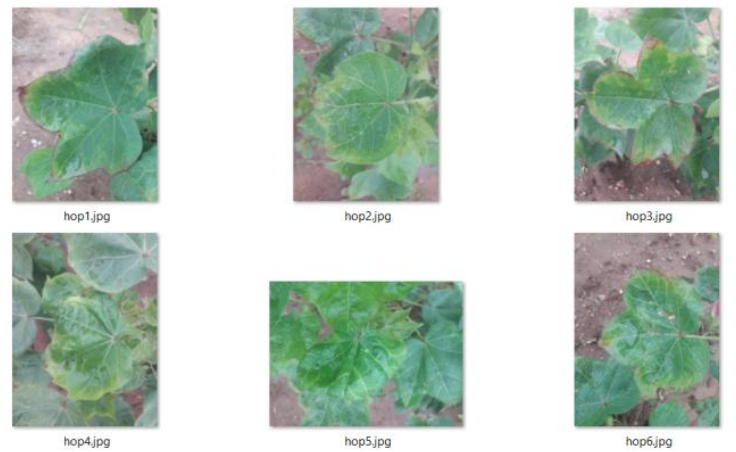

Figure.3 Images of Leaf hopper (training and test image) 


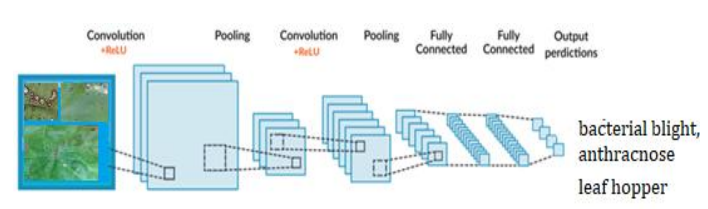

Figure.4 CNN architecture for cotton disease diagnosis

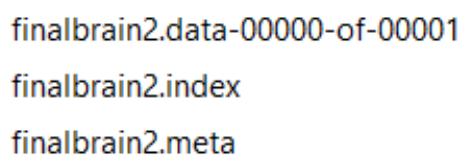

Figure.5 Model generated for cotton disease using Python programming

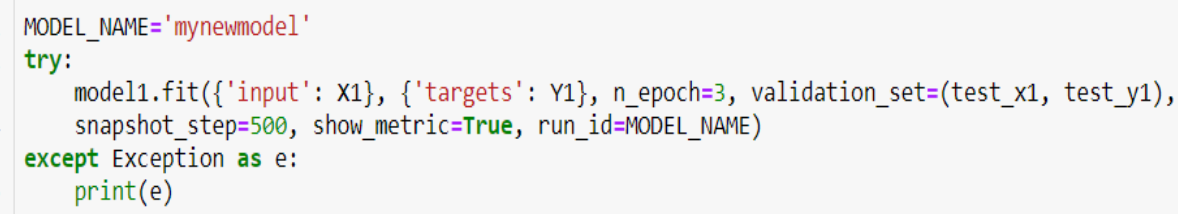

Training Step: 494 | total loss: 0.20240 | time: $134.195 \mathrm{~s}$

| Adam | epoch: 003 | loss: 0.20240 - acc: 0.9338 -- iter: 10496/10542

Training Step: 495 | total loss: 0.19157 | time: $136.354 \mathrm{~s}$

| Adam | epoch: 003 | loss: 0.19157 - acc: 0.9389 | val_loss: 0.26688 - val_acc: 0.9040 -- iter: 10542/10542

Figure.6 Accuracy of CNN model

\section{CNN performance}

CNN Model is evaluated from the image captured from the cotton field to check the accuracy of the model. The accuracy of the model is $93.89 \%$ as shown in Figure 6 with the training and test images. The model is also tested with the real time images captured from the cotton field.

In conclusion, cotton crop is cultivated for its fiber, which is commonly used as a textile raw material across the world. In the current paper, CNN-based research efforts applied in the cotton disease diagnosis. The model examined the particular area and problem ie., the leaf disease in cotton, listed technical details of the models, described about the sources of images used and reported the overall accuracy achieved. The accuracy of the model is $93.89 \%$ with the training and test images. To construct $\mathrm{CNN}$, a large number of training data sets are very much essential to get the accurate result. So in future the dataset is expanded to improve the accuracy of the model. For future work, it is planned to capture all the cotton diseases in stem, boll and leaves.

\section{References}

Alex K., I. Sutskever and Hinton G. E., 2012. Imagenet classification with deep convolutional neural networks. Neural Information Processing Systems (NIPS). Pp. 1097-1105.

Boulent Justine, Foucher Samuel, Théau Jérôme, St-Charles Pierre-Luc, 2019. Convolutional Neural Networks for the Automatic Identification of Plant Diseases. Frontiers in Plant Science. 10, 1-15

Food and Agriculture Organization of the United Nation, International Plant Protection Convention, Plant Health and Food Security. Pamphlet 
I7829EN/1/09.17 (accessed April 04, 2019)

Gill, H., and Garg, H., 2014. Pesticides: environmental impacts and management strategies in Pesticides-Toxic Effects eds S. Solenski and M. L. Larramenday (Rijeka: InTech). Pp. 188-230.

Goulson, D., 2014. Ecology pesticides linked to bird declines. Nature. 511. 295- 296.

Knillmann, S., and Liess, M., 2019. Pesticide Effects on Stream Ecosystems. Cham: Springer International Publishing, Pp. $211-214$.

Liu, Z., J. Gao, G. Yang, H. Zhang, and Y. $\mathrm{He}$ 2016. Localization and Classification of Paddy Field Pests using a Saliency Map and Deep Convolutional Neural Network. Scientific Reports, 6, 1-12.

Risebrough R.W., 1986. Pesticides and Bird Populations, In: Johnston R.F. (eds)
Current Ornithology, 3, 397-427.

Sanchez-Bayo F, Goka K, 2014. Pesticide Residues and Bees - A Risk Assessment. PLoS ONE, 9, 1-16.

Sladojevic S., Arsenovic M., Anderla A., Culibrk D., and Stefanovic D., 2016, Deep neural networks based recognition of plant diseases by leaf image classification, Computational Intelligence and Neuroscience, Pp. 111.

Weisenburger, D. D., 1993, Human health effects of agrichemical use, Human Pathol. 24, 571-576

Yalcin H. and Razavi S., 2016. Plant classification using convolutional neural networks, Fifth International Conference on Agro-Geoinformatics (Agro-Geoinformatics), Tianjin. Pp. 15 .

\section{How to cite this article:}

Kalpana, M., K. Senguttuvan and Latha, P. 2020. Automatic Pest Identification for Cotton Crop Using Convolution Neural Network. Int.J.Curr.Microbiol.App.Sci. 9(09): 2112-2117. doi: https://doi.org/10.20546/ijcmas.2020.909.263 